\title{
Disability from posttraumatic headache is compounded by coexisting posttraumatic stress disorder
}

This article was published in the following Dove Press journal:

Journal of Pain Research

2I August 2017

Number of times this article has been viewed

\author{
Louise S Roper ${ }^{1-3}$ \\ Peter Nightingale ${ }^{4}$ \\ Zhangjie $\mathrm{Su}^{5,6}$ \\ James L Mitchell ${ }^{1-3}$ \\ Antonio Belli5,6 \\ Alexandra J Sinclair ${ }^{1-3}$ \\ 'Institute of Metabolism and \\ Systems Research, College of \\ Medical and Dental Sciences, \\ University of Birmingham, ${ }^{2}$ Centre \\ for Endocrinology, Diabetes and \\ Metabolism, Birmingham Health \\ Partners, ${ }^{3}$ Department of Neurology, \\ ${ }^{4}$ Wolfson Computer Laboratory, \\ University Hospital Birmingham \\ NHS Foundation Trust, ${ }^{5}$ Institute of \\ Inflammation and Ageing, College \\ of Medical and Dental Sciences, \\ University of Birmingham, ${ }^{6} \mathrm{Health}$ \\ Research Surgical Reconstruction \\ and Microbiology Research Centre, \\ Birmingham, UK
}

Background: Posttraumatic headache (PTH) occurs in up to $82 \%$ of patients with traumatic brain injury (TBI). Posttraumatic stress disorder (PTSD) occurs in 39\% of those with PTH. This study evaluates whether PTSD affects PTH disability.

Methods: Eighty-six patients with TBI were prospectively evaluated in a secondary care trauma center. Headache disability was assessed using the Headache Impact Test version 6 and signs indicative of PTSD using the PTSD Check List Civilian version.

Results: Increased PTSD-type symptoms were significantly associated with increased headache disability ( $p<0.001$ ), as were employment status and loss of consciousness ( $p=0.049$ and 0.016 , respectively). Age was negatively correlated with headache disability (Spearman's correlation rho $=0.361, p=0.001)$.

Conclusion: Increased severity of PTSD-type symptoms is significantly associated with increased headache disability in patients with chronic PTH. Managing PTSD symptoms in patients with chronic PTH may facilitate headache management.

Keywords: chronic headache, traumatic brain injury, neurotrauma

\section{Introduction}

PTH is defined as a headache with onset within 7 days of head trauma or within 7 days of regaining consciousness following head trauma. ${ }^{1}$ It has been reported to occur in $37 \%-82 \%$ of patients with TBI. ${ }^{2-4}$ Of these headaches, $47 \%-95 \%$ will persist for $>3$ months and are defined as chronic PTH. ${ }^{5}$ Chronic PTH is often persistent over at least 12 months, ${ }^{2,6}$ with $>40 \%$ of patients with TBI reporting disability that persists at 1 year post-injury. ${ }^{7}$ Phenotypically, PTH resembles migraine in $49 \%$ of cases and tension-type headache in $40 \%$, whereas $11 \%$ are undefined. ${ }^{6}$ Migraine is the seventh most disabling disease worldwide, and migraine following TBI is a major cause of disability. ${ }^{8}$ Subjects with new-onset PTH have decreased ability in memory tasks, slower reactions, and struggle in returning to their previous physical and social functions following TBI. Thus, PTH has a profound emotional, social, and economic impact. ${ }^{3,9,10}$

Headache disability following trauma may be worsened by the presence of comorbid PTSD and has already been documented as more pronounced in those with preexisting primary headaches. ${ }^{9-11}$ Pre-injury migraine may be the greatest predictor of PTH frequency and severity, ${ }^{4,6,7,12}$ with chronic migraineurs being at greater risk than those with episodic migraine. ${ }^{13}$ Gender, age, and severity of TBI have also been shown to influence PTH., 4,6,711,12,14 Gender and severity of injury interact and have a complex
Correspondence: Louise S Roper Institute of Metabolism and Systems Research, College of Medical and Dental Sciences, University of Birmingham, Edgbaston, West Midlands, Birmingham BI5 2TT, UK

Email louisesarah.roper@nhs.net 
effect on PTH. In the case of mild TBI, there is no difference in the incidence of PTH between men and women; ${ }^{6}$ however, PTH is reported to be more prevalent and persistent in women who have suffered moderate/severe injuries. ${ }^{4,7,11,12}$ Women may have greater headache-associated disability than men at 12 months, ${ }^{4,7}$ and differences in reporting of PTH between men and women persist at 11 years postinjury. ${ }^{12}$ Older patients have less incidence of PTH, but this may represent underreporting in this age group ${ }^{6}$ and hence requires further investigations.

Patients who have suffered TBI may have symptoms of PTSD. PTSD is a syndrome defined by the DSM-IV as a disorder related to actual or perceived harm to the individual or a close relative/friend or repeated exposure to aversive details of a traumatic event. Symptoms include intrusive memories, distressing dreams, flashbacks, psychological distress, and physiological adverse reactions. ${ }^{15}$ Reported prevalence of PTSD symptoms following TBI varies widely from $6 \%$ to $39 \%$ across both military and civilian populations. ${ }^{3,12,16}$ Headache may hinder recovery from the cognitive and behavioral consequences of TBI. ${ }^{6}$ Patients frequently have both PTH and PTSD symptoms, ${ }^{3,6,12,16}$ but the relationship between the symptoms is less defined.

In this study, we primarily hypothesize that headache disability following TBI is worsened by PTSD. Furthermore, we look at other factors that are associated with headache disability post-TBI.

\section{Methods}

Consecutive patients with a history of TBI were prospectively seen in the TBI outpatient clinic at University Hospital Birmingham NHS Foundation Trust, UK, a secondary care trauma center, from December 2012 to July 2014. All patients with a TBI were eligible for this study. Patients were excluded if they failed to complete the headache or PTSD questionnaires. Ethical approval was given for the collection and use of these data by the Research and Audit Department of the University Hospital Birmingham, Queen Elizabeth. Verbal informed consent was obtained from all participants in this study. Data were collected over 19 months, but the data for each individual related to a single time point. Although informed consent was obtained from patients in order to collect these data and analyze them, express permission was not obtained to make the raw data public. The datasets are anonymous and can be provided as additional files if requested.

Headache disability was assessed using the Headache Impact Test version 6 (HIT-6). ${ }^{17}$ Scores were categorized into the following groups: severe impact (score $\geq 60$ ), substantial impact (score 56-59), limited impact (score 50-55), and little to no impact (score $\leq 49$ ). ${ }^{17}$ PTSD symptoms were assessed using PTSD Check List Civilian version (PCL-C). ${ }^{18,19}$ A score of $>30$ indicates the presence of PTSD symptoms. ${ }^{20} \mathrm{PCL}-\mathrm{C}$ is sanctioned by the National Institute for Health and Care Excellence for use in evaluating PTSD symptoms. ${ }^{21}$ It was chosen for use in this study due to its relatively few number of items and simple scoring system, which increases its reliability. ${ }^{22}$

Other factors investigated were the following: gender; educational level (high school or university); ethnicity; employment status (categories based on those used by the Office of National Statistics, $\mathrm{UK}^{23}$ ); interval from admission to hospital to filling in the questionnaire; whether an LOC occurred, either at the time of injury or subsequently up to and including the time of admission; whether seizures occurred, either associated with the injury or within 24 hours post-injury; GCS on admission (a score of $<13$ at admission defined a moderate/severe TBI); ${ }^{1,24}$ GOS; ${ }^{24}$ whether the patient was intoxicated with alcohol at the time of the injury; MOI (fall, road traffic accident, attack, home accident, selfharm, sports injury, or unknown mechanism); and severity of the injury on neuroimaging, quantified by the Marshall grading of patient's first CT scan (scoring 1-6, with 1 being minimal injury and 6 being considerable). ${ }^{25}$

Statistical analyses were undertaken on IBM SPSS (version 20.0, released 2011). Data were not normally distributed, with the exception of age; therefore, nonparametric tests were employed in the main analysis. HIT-6 and PCL-C scores (noncategorical data) were assessed using Kruskal-Wallis and Mann-Whitney U-tests as appropriate. Spearman's correlations were used to quantify the association between HIT-6 and PCL-C scores. HIT-6 and PCL-C categories (as described earlier) were used for graphical representation but not for statistical analysis. A Bonferroni correction was applied to account for multiple analyses. Data were further evaluated using a multiple linear regression model. Statistical significance was considered at $p \leq 0.05$.

\section{Results}

In total, 151 consecutive patients with TBI were approached in a TBI clinic. Twelve patients were not recruited due to the inability to engage with the study. The remaining 139 patients were recruited, but 53 were excluded because they did not complete the headache and PTSD questionnaires, which reflect the primary aims of this study. Therefore, data from 86 patients were analyzed (Figure 1). The majority of our cohort were white males $(n=65,75.6 \%)$ aged $45 \pm 20$ years. Patients in this study were evaluated at a median time 


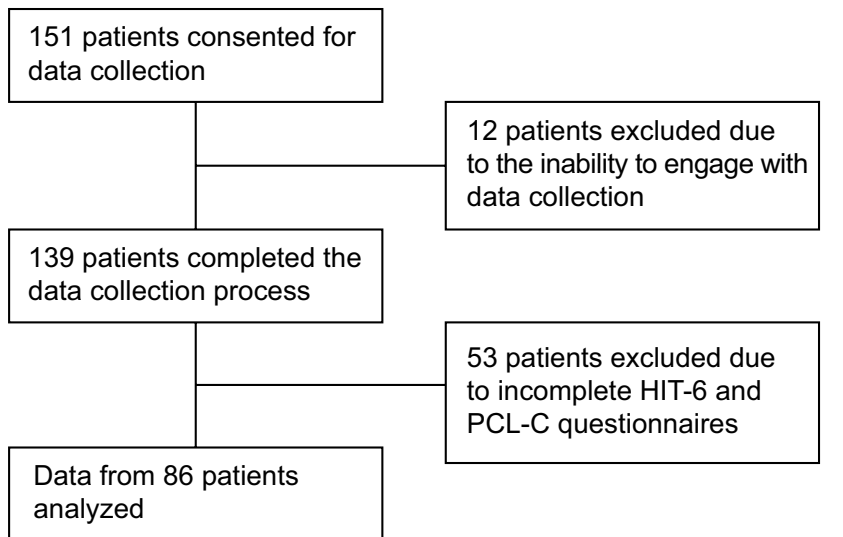

Figure I Diagram showing the selection of study patients.

Abbreviations: HIT-6, Headache Impact Test version 6; PCL-C, PTSD Check List Civilian version; PTSD, posttraumatic stress disorder.

of 5.6 months (170 days) post-injury in an outpatient clinic. Results are displayed in Table 1 and discussed further.

Fifty-five patients (64\%) had a mild TBI, and 21 patients (24\%) had suffered moderate/severe TBIs (data on severity of injury were not recorded in 10 participants). The median HIT-6 score was 46 (range 36-78). Fifty-one patients (59\%) suffered "little or no impact" from their headache; 35 patients (41\%) had PTHs, and of these, 25 patients (29\%) suffered "severe or substantial impact" as defined by the HIT-6 score (Figure 2A).

Of the 21 patients who sustained moderate/severe TBI, seven $(33 \%)$ suffered a severe impact from their PTH, whereas of the 55 patients with mild TBI, only $10(18 \%)$ suffered a severe impact of headache (Figure 2A). Consequently, we found that there was a trend to a greater severity of headache disability in those with a more severe head injury.

The median PCL-C score was 26 (range 17-79). In our study, 35 participants (41\%) had a PCL-C score that indicated a diagnosis of PTSD. Among those with substantial or severe headache disability (HIT-6 score $>56$ ), the majority also had PTSD (72\%). Among those with low headache disability (HIT-6 score <49), only 24\% had PTSD (Figure 2B).

Thirty participants (35\%) had discontinued full-time education after high school, 29 (34\%) were employed, 12 (14\%) were retired, and 12 were students.

Thirty three patients $(38 \%)$ suffered a LOC, either at the time of the injury or on admission. The median GCS on presentation was 14 (range 3-15), the median GOS was 5 (range $3-5$ ), and only four patients $(<5 \%)$ had a seizure. The median Marshall grade of the CTs of these patients was 2 (range 1-6, mode 2). Falls were the most common mechanism of TBI (34 patients; $40 \%$ ) in this study.

We assessed factors that were associated with headache disability. PTSD was significantly correlated with headache disability (HIT-6; rho=0.583, $p<0.001$; Figure 3 ). Moreover,
Table I Participant demographics

\begin{tabular}{|c|c|}
\hline Demographics & n (\%) \\
\hline \multicolumn{2}{|l|}{ Gender } \\
\hline Male & $65(76)$ \\
\hline Age* & $45(20)$ \\
\hline \multicolumn{2}{|l|}{ Education } \\
\hline High school & $30(35)$ \\
\hline University & $12(14)$ \\
\hline Unknown & $44(5 \mathrm{I})$ \\
\hline \multicolumn{2}{|l|}{ Ethnicity } \\
\hline White British & $65(76)$ \\
\hline South Asian & $5(6)$ \\
\hline Black and white mixed Caribbean & $\mathrm{I}(\mathrm{I})$ \\
\hline African & $\mathrm{I}(\mathrm{I})$ \\
\hline Oriental Asian & I (I) \\
\hline Unknown & $13(15)$ \\
\hline \multicolumn{2}{|l|}{ Employment status } \\
\hline Professional & $10(12)$ \\
\hline Retired & $12(14)$ \\
\hline Semiprofessional & $6(7)$ \\
\hline Skilled laborer & $13(15)$ \\
\hline Student & $12(14)$ \\
\hline Unemployed & II (I3) \\
\hline Unknown & $22(26)$ \\
\hline Interval from admission to completing questionnaire ${ }^{\mathrm{T}}$ & $170(16-1305)$ \\
\hline Loss of consciousness & $33(38)$ \\
\hline Seizure & $4(5)$ \\
\hline Glasgow Coma Scale ${ }^{\mathrm{T}}$ & $14(3-15)$ \\
\hline Glasgow Outcome Scale ${ }^{\mathrm{T}}$ & $5(3-5)$ \\
\hline Alcohol consumption & $21(24)$ \\
\hline \multicolumn{2}{|l|}{ Mechanism of injury } \\
\hline Fall & $34(40)$ \\
\hline Road traffic accident & $23(27)$ \\
\hline Attack & $2(2)$ \\
\hline Home accident & $20(23)$ \\
\hline Self-harm & $\mathrm{I}(\mathrm{I})$ \\
\hline Sports injury & $3(4)$ \\
\hline Unknown & $3(4)$ \\
\hline Marshall CT grade ${ }^{\mathrm{F}}$ & $2(I-6)$ \\
\hline I & 14 \\
\hline 2 & 42 \\
\hline 3 & 6 \\
\hline 4 & 2 \\
\hline 5 & 2 \\
\hline 6 & 12 \\
\hline Unknown & 8 \\
\hline \multicolumn{2}{|l|}{ Severity of injury ${ }^{*}$} \\
\hline Mild & $55(64)$ \\
\hline Moderate/severe & $21(24)$ \\
\hline Unknown & $10(12)$ \\
\hline HIT-6 score ${ }^{\mathrm{F}}$ & $46(36-78)$ \\
\hline Little or no impact & $5 \mathrm{I}(59)$ \\
\hline Limited impact & $10(12)$ \\
\hline Substantial impact & $5(6)$ \\
\hline Severe impact & $20(23)$ \\
\hline PCL-C score ${ }^{\mathrm{F}}$ & $26(17-79)$ \\
\hline Positive PTSD diagnosis & $35(4 I)$ \\
\hline
\end{tabular}

Notes: *Age: mean and standard deviation are shown. ${ }^{\mathrm{T}}$ Median and range are shown for interval from admission to completion of questionnaire, Glasgow Coma Scale, Glasgow Outcome Scale, Marshall CT grade, HIT-6 score, and PCL-C score. -As defined by the International Classification of Headache (Beta 3 version).

Abbreviations: CT, computed tomography; HIT-6, Headache Impact Test version 6; PCL-C, PTSD Check List Civilian version; PTSD, posttraumatic stress disorder. 

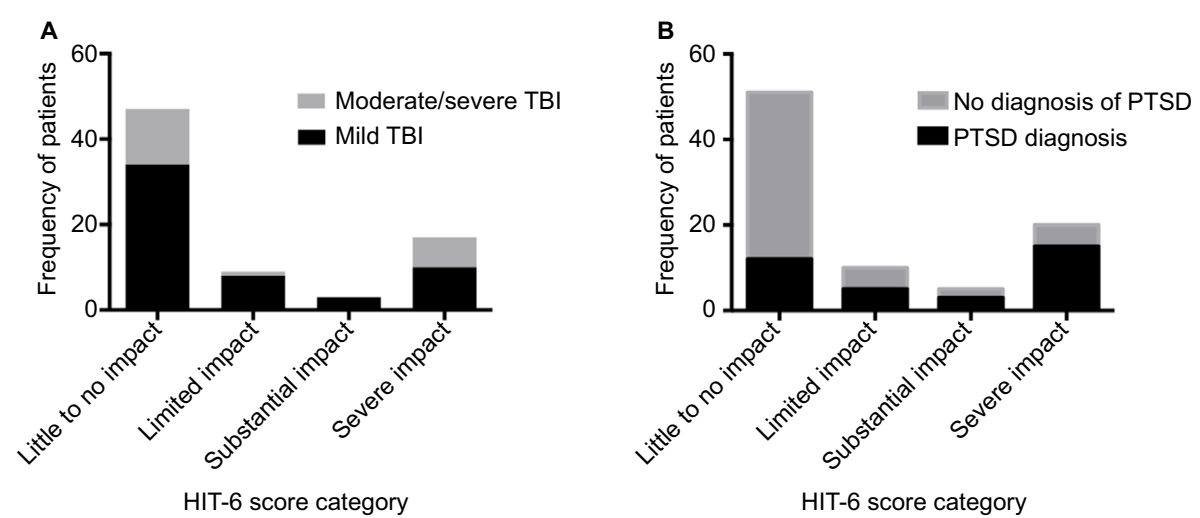

Figure 2 The number of patients with little, limited, substantial, and severe impact from PTH, as defined by their HIT-6 score. (A) Subcategorization by the number of patients with mild or moderate/severe TBI. (B) Subcategorization by the number of patients whose PCL-C scores indicate the presence/absence of PTSD symptoms. Abbreviations: PTH, posttraumatic headache; HIT-6, Headache Impact Test version 6; TBI, traumatic brain injury; PCL-C, PTSD Check List Civilian version; PTSD, posttraumatic stress disorder.

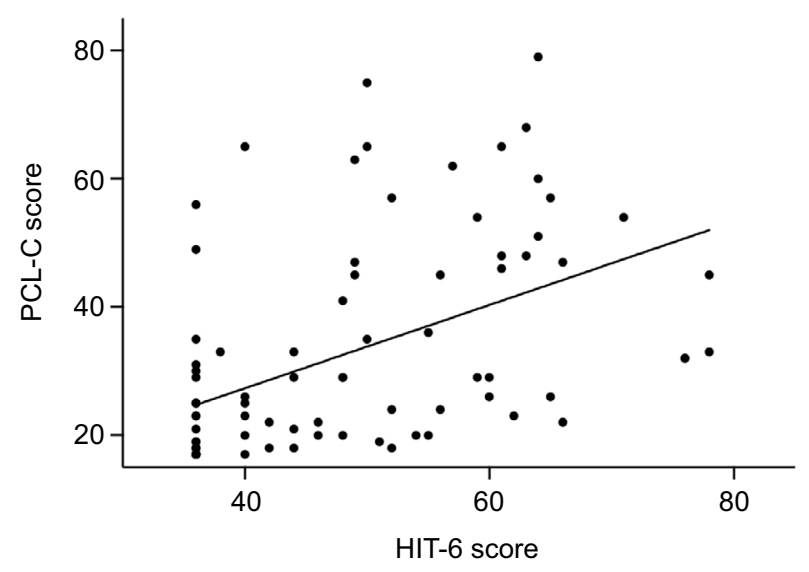

Figure 3 HIT- 6 and PCL-C scores correlated significantly (rho=0.583, $p<0.00 \mathrm{I}$ ). Abbreviations: HIT-6, Headache Impact Test version 6; PCL-C, PTSD Check List Civilian version; PTSD, posttraumatic stress disorder.

LOC was associated with headache disability $(p=0.016)$. Age was negatively correlated with HIT-6 scores (rho $=-0.361$, $p=0.001$ ). Employment status was significantly associated with both headache disability and PTSD symptoms ( $p=0.049$ and 0.046 , respectively). MOI was associated with PTSD symptoms $(p=0.021)$, but not headache disability. No significant association between headache disability and the following factors was found: gender, educational level, ethnicity, interval from admission to completion of the questionnaire, seizures, GOS, presence of alcohol consumption, MOI, and Marshall CT grade. After applying a Bonferroni correction, only age and PTSD were significantly correlated with headache disability. Data were further evaluated in a multiple linear regression model, which highlighted that it was predominantly the PCL-C score $(p<0.001)$ that explained the variability in the HIT- 6 score. Furthermore, age was a secondarily significant variable in the multiple linear regression model $(p=0.002)$.

\section{Discussion}

In this study of patients with TBI, we primarily evaluated the relationship between headache disability and PTSD symptoms. In addition, we analyzed patient demographics and variables at the time of injury. Patients in this study were evaluated at a median time of 5.6 months (170 days) post-injury, which is consistent with the diagnosis of chronic PTH (defined as a PTH continuing after 3 months). ${ }^{1}$ As hypothesized, we found that, in our cohort of patients, the factor most significantly associated with headache disability was the presence of comorbid PTSD symptoms. This is in keeping with studies of military patients where the Migraine Disability Assessment Score has been found to be associated with the presence of PTSD, despite these military patients sustaining more severe head injuries than patients in our cohort (64\% LOC vs 38\% LOC in our cohort). ${ }^{3}$ Our study identifies a comorbidity prevalence rate of $40 \%$ for PTSD symptoms and PTH, which concurs with the results reported from another recent work. ${ }^{9}$

Contrary to the findings of Walker et al, ${ }^{4}$ we did not find any association between gender and PTH severity. Despite having a similar gender distribution as our study, the study by Walker et al showed that women reported headache symptoms with 1.89 times more severity than men. ${ }^{4}$ However, Walker et al used a different tool to calculate the "headache density", whereas we quantified the disability associated with our cohort's headaches. ${ }^{17}$ Similarly, Ahman et al found that PTSD was more common in women than in men, and that the grading of women's symptoms was likely to be higher than that of men's symptoms. ${ }^{12}$ This was not the case in our study cohort as PCL-C scores were evenly distributed across the genders.

Furthermore, our additional analyses highlighted that age impacted on PTH. This is in agreement with the results reported by Lucas et $\mathrm{al}^{6}$ who found that patients older than 60 
years were significantly less likely to report PTH. The mean age of their cohort and ours is similar (44-46 and 45 years, respectively). ${ }^{6}$ Building on this finding, we show that older age correlated significantly with decreased PTH severity. To ascertain why this is the case, further work is needed; however, we suggest that it may be because primary headaches are also less common in the elderly ${ }^{26}$ or because of lower reporting of headaches in the elderly.

This study is limited by the collection of data at a single time point, and future studies evaluating longitudinal changes will be valuable. Moreover, it is important to understand that although we have identified that the degree of headache disability after brain trauma is associated with PTSD symptoms, this does not imply causation; however, it would be of interest to evaluate whether treatment of PTSD improves headache disability. Furthermore, it would have been interesting to note familial and prior history of migraine, although this was not the primary focus of this study. In future research, a collection of prospective headache diaries, which includes monitoring of headache frequency and severity over time in relation to severity of PTSD symptoms, would be valuable.

\section{Conclusion}

We demonstrate, in a population with predominant mild TBI, that headache disability is greatest in those with PTSD symptoms. This is in agreement with studies suggesting that emotional health can impact headache disability. ${ }^{27}$ Moreover, headache disability is increased in individuals who are younger or lose consciousness at the time of injury. We suggest that in patients with chronic PTH, identifying and treating PTSD may be beneficial for headache management.

\section{Clinical implications}

- Patients with chronic PTH have greater headache disability if they also have PTSD.

- Younger patients who lose consciousness at the time of injury may have the most disability from chronic PTH.

- In patients with chronic PTH and PTSD, headache disability might be improved if PTSD is actively managed alongside headache management.

\section{Abbreviations}

CT, computed tomography

DSM-IV, Diagnostic and Statistical Manual of Mental Disorders, version IV

GCS, Glasgow Coma Scale

GOS, Glasgow Outcome Scale

HIT-6, Headache Impact Test version 6
LOC, loss of consciousness

MOI, mechanism of injury

PCL-C, PTSD Check List Civilian version

PTH, posttraumatic headache

PTSD, posttraumatic stress disorder

TBI, traumatic brain injury

\section{Acknowledgments}

The authors thank Ahmed Al-Jabir for providing technical support.

\section{Author contributions}

Louise Roper contributed to data cleaning, data coding and analysis, and writing of the article. Peter Nightingale contributed to statistical analysis. Zhangjie Su contributed to study design, data collection, and review of the article. James Mitchell contributed to review and writing of the article. Antonio Belli contributed to study design and data collection. Alexandra J Sinclair contributed to writing of the article. All authors contributed toward data analysis, drafting and critically revising the paper and agree to be accountable for all aspects of the work.

\section{Disclosure}

Dr Alexandra J Sinclair was financially supported by an NIHR Clinician Scientist Fellowship (NIHR-CS-011-028) and by the Medical Research Council, UK (MR/K015184/1). Professor Antonio Belli and Dr Zhangjie Su were financially supported by the National Institute for Health Research Surgical Reconstruction and Microbiology Research Centre. The views expressed are those of the authors and not necessarily those of the National Health Service, the NIHR or the Department of Health. The authors report no other conflicts of interest in this work.

\section{References}

1. Headache Classification Committee of the International Headache Society (IHS). The International Classification of Headache Disorders, 3rd edition (beta version). Cephalalgia. 2013;33(9):629-808.

2. Lew HL, Otis JD, Tun C, et al. Prevalence of chronic pain, posttraumatic stress disorder, and persistent postconcussive symptoms in OIF/OEF veterans: polytrauma clinical triad. J Rehabil Res Dev. 2009;46(6):697-702.

3. Rosenthal JF, Erickson JC. Post-traumatic stress disorder in U.S. soldiers with post-traumatic headache. Headache. 2013;53(10):1564-1572.

4. Walker WC, Marwitz JH, Wilk AR, et al. Prediction of headache severity (density and functional impact) after traumatic brain injury: a longitudinal multicenter study. Cephalalgia. 2013;33(12):998-1008.

5. Defrin R. Chronic post-traumatic headache: clinical findings and possible mechanisms. J Man Manip Ther. 2014;22(1):36-44.

6. Lucas S, Hoffman J, Bell K, et al. Characterization of headache after traumatic brain injury. Cephalalgia. 2012;32(8):600-606. 
7. Hoffman JM, Lucas S, Dikmen S, et al. Natural history of headache after traumatic brain injury. J Neurotrauma. 2011;28(9):1719-1725.

8. Steiner TJ, Stovner LJ, Birbeck GL. Migraine: the seventh disabler. J Headache Pain. 2013;14(1):1.

9. Kjeldgaard D, Forchhammer H, Teasdale T, et al. Chronic post-traumatic headache after mild head injury: a descriptive study. Cephalalgia. 2014;34(3):191-200.

10. Monteith T, Borsook D. Insights and advances in post-traumatic headache: research considerations. Curr Neurol Neurosci Rep. 2014;14(2):428.

11. Mihalik JP, Register-Mihalik J, Kerr ZY, Marshall SW, McCrea MC, Guskiewicz KM. Recovery of posttraumatic migraine characteristics in patients after mild traumatic brain injury. Am J Sports Med. 2013;41(7):1490-1496.

12. Ahman S, Saveman BI, Styrke J, Björnstig U, Stålnacke BM. Long-term follow-up of patients with mild traumatic brain injury: a mixed-method study. J Rehabil Med. 2013;45(8):758-764.

13. Peterlin BL, Tietjen G, Meng S, Lidicker J, Bigal M. Post-traumatic stress disorder in episodic and chronic migraine. Headache. 2008; 48(4):517-522.

14. Gladstone J. From psychoneurosis to ICHD-2: an overview of the state of the art in post-traumatic headache. Headache. 2009;49(7):1097-1111.

15. Hull AM, Curran SA. Be vigilant for post-traumatic stress reactions. Practitioner. 2016;260(1793):19-23.

16. Cifu DX, Taylor BC, Carne WF, et al. Traumatic brain injury, posttraumatic stress disorder, and pain diagnoses in OIF/OEF/OND Veterans. J Rehabil Res Dev. 2013;50(9):1169-1176.

17. Yang M, Rendas-Baum R, Varon SF, Kosinski M. Validation of the Headache Impact Test (HIT-6 $\left.6^{\mathrm{TM}}\right)$ across episodic and chronic migraine. Cephalalgia. 2011;31(3):357-367.

18. Smith M, Redd W, Duhamel K, Vickberg SJ, Ricketts P. Validation of the PTSD Checklist-Civilian Version in survivors of bone marrow transplantation. J Trauma Stress. 1999;12(3):485-499.
19. Blanchard EB, Jones-Alexander J, Buckley TC, Forneris TC. Psychometric properties of the PTSD Checklist (PCL). Behav Res Ther. 1996;34(8):669-673.

20. Using the PTSD Checklist for DSM-IV (PCL). 2015. Available from: https://www.ptsd.va.gov/professional/assessment/documents/ PCL_handoutDSM4.pdf. Accessed June 6, 2017.

21. National Collaborating Centre for Mental Health (UK). Post-Traumatic Stress Disorder: The Management of PTSD in Adults and Children in Primary and Secondary Care. NICE Clinical Guidelines No. 26. Leicester: Gaskell; 2005.

22. Brewin CR. Systematic review of screening instruments for the detection of posttraumatic stress disorder in adults. $J$ Trauma Stress. 2005;18(1):53-62.

23. SOC2010 volume 1: structure and descriptions of unit groups. 2015. Available from: http://www.ons.gov.uk/ons/guide-method/classifications/current-standard-classifications/soc2010/soc2010-volume1-structure-and-descriptions-of-unit-groups/index.html. Accessed December 19, 2015.

24. Bigler E, Maxwell W. Neuropathology of mild traumatic brain injury: relationship to neuroimaging findings. Brain Imaging Behav. 2012;6(2):108-136.

25. Watadani T, Sakai F, Johkoh T, et al. Interobserver variability in the CT assessment of honeycombing in the lungs. Radiology. 2013; 266(3):936-944.

26. Martins KM, Bordini CA, Bigal ME, Speciali JG. Migraine in the elderly: a comparison with migraine in young adults. Headache. 2006;46(2):312-316.

27. Abu Bakar N, Tanprawate S, Lambru G, Torkamani M, Jahanshahi M, Matharu M. Quality of life in primary headache disorders: a review. Cephalalgia. 2016;36(1):67-91.
Journal of Pain Research

\section{Publish your work in this journal}

The Journal of Pain Research is an international, peer reviewed, open access, online journal that welcomes laboratory and clinical findings in the fields of pain research and the prevention and management of pain. Original research, reviews, symposium reports, hypothesis formation and commentaries are all considered for publication.

\section{Dovepress}

The manuscript management system is completely online and includes a very quick and fair peer-review system, which is all easy to use. Visit http://www.dovepress.com/testimonials.php to read real quotes from published authors. 